\title{
Using Finite Element Analysis Methods to Reduce the Failure of Building Structures
}

\author{
Daniel J. Thomas
}

Submitted: 16 May 2020/Published online: 2 June 2020

(C) ASM International 2020

When developing modern building structures, it is necessary to ensure that integrity and long-term reliability is achieved and that it is uncompromised throughout the entire life of the structure [1]. Previously, structural engineers would progress from a computer-aided design and then perform hand stress calculations. At this stage in the design process, if the structure is determined to be structurally insufficient, then this can require redesign and subsequently further calculations performed [2]. This can lead to the development of numerous iterative designs that can often result in a higher number of engineering design hours, subsequent additional costs incurred, time delays and non-optimum designs produced.

The drive towards the fabrication in efficient building designs that achieve ambitious architectural design requirement often poses a challenge for structural engineers. This is particularly the case with landmark projects. Therefore, a monumental opportunity has progressed in the form of finite element analysis (FEA). This is used to design a building from the ground up, form the foundations, the super-structure and substructure elements. This also incorporates libraries of every bolt, washer and cleat structural steel system, together with the structural fabrication standards used across the different countries around the World.

During the sequential design engineering and stress analysis process, a complex building assembly can be designed safely, imaginatively and holistically with reduced chance of structural failure. This is particularly the case of the final structure being optimised at its location,

D. J. Thomas $(\varangle)$

Creation Engineering Group, Llynfi Enterprise Centre, Bridgend, UK

e-mail: daniel.thomas@creationengineering.co.uk ground conditions and complex loading patterns. For structural engineers, FEA design is critical for identifying possible design flaws in all the complex structural elements. This is particularly the case when a design is modified continuously by the architect or where modifications have been requested by the contractor.

This has been particularly found in a project that I recently worked on as shown in Fig. 1. When designing a large warehouse project, particular issues were determined with future weather conditions due to climate change. The structure has a large roof profile and was a four-story structure. This makes the probability of this building experiencing higher winds and the potential for extensive snow loading over the next 40 years. The structure consisted of over two thousand beams, channels, columns and braces. However, by using FEA software and automated tools this project was designed to a high degree of accuracy. With the complex structural assembly being designed down to the last bolt, washer and weld. In this way, FEA is used to consider how the structure will respond to complex loading regimes and to predict the probability of structural failure over extended periods of time [3]. Each of these factors is used to develop different scenarios, that can each be assessed in detail. The types of loads that are assessed for a building structure include:

1. Dead loads

2. Imposed loads

3. Wind loads

4. Special loads

5. Snow loads

6. Seismic loads

During the computational modelling phases of FEA, the assembly is modelled with fixed, pin and sliding constraints as it would experience in real life. This is applied together 
Fig. 1 Warehouse structural project designed by the author using Autodesk Advance Steel and Robot structural analysis used to optimise the performance of the building structure

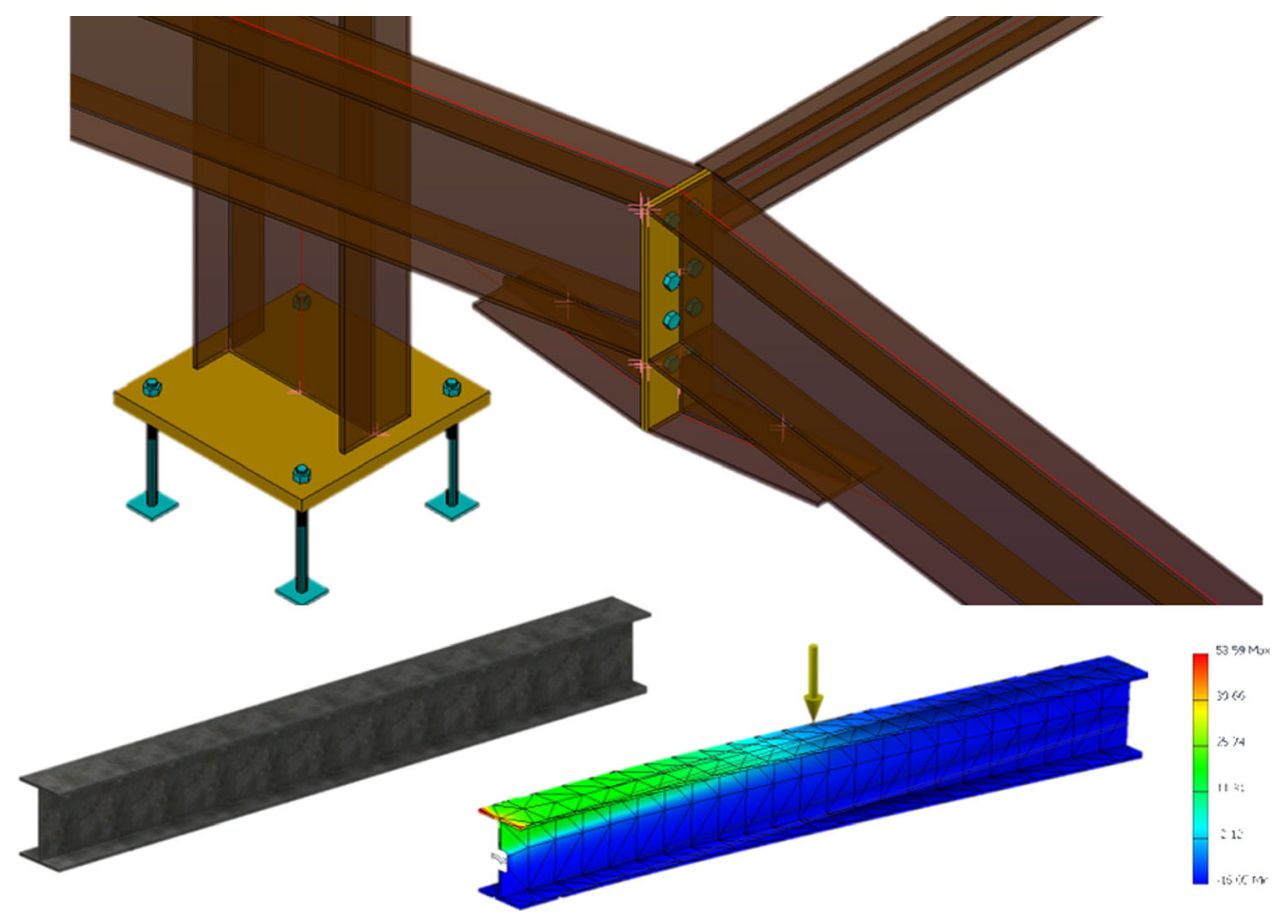

with the different loads experienced at each element of the structure. The assembly is then divided into many millions of geometrical elements and interconnecting nodes, each of which is subjected to a mathematical calculation. The software pre-processor then accumulates each element of data that performs a sequence of calculations that demonstrate how the structure will respond to structural loading. The regions that display the highest level of stress and/or displacement require a higher level of element mesh refinement.

Under each loading condition, FEA software predicts if the component will fracture, distort or buckle and by how much when subjected to different loading scenarios. It is used to demonstrate that a design will work according to the design requirements for rigidity, safety and displacement under load. The benefits of using FEA approaches for minimising failure include:

- Assessment of and assurance of the product safety factor, which is usually to an order of magnitude over the minimum safety factor and yield strength of the selected structural elements.

- Determining improvements in the structures design, including optimising strength and enhancing long-term durability, while simultaneously enhancing the formation and positioning of complex structural elements.

- Increased confidence in the design work and that it will perform as intended when the structure is built and undergoing complex loading patterns.

It is this level of detail that can be used to assess the performance of every beam, column and cross-brace which provides an accurate representation of the real-world performance. For instance, using different Rolled Steel Joist (RSJ) types in a design can have a significant effect on the stiffness and loading properties as can the position of crossbraces and how the structure is fixed together.

Over recent years, I have found that FEA software tools reduce the reliance on traditional hand calculations. However, although these software tools provide an optimum way to analyse, optimise and engineer complex building structures, hand calculations still provide a means to confirm that FEA results are correct. Computer-based analysis of building structures is a vital tool to evaluate and subsequently prevent the failure of structures. This is particularly the case with complex architectural design challenges and ground constraints that can be encountered. FEA can be used by all structural engineers to prevent the failure of a design and optimise the time that it takes to develop a durable design on a daily basis.

\section{References}

1. H.-S. Kim, Y.-J. Lim, H.-L. Lee, Optimum location of outrigger in tall buildings using finite element analysis and gradient-based optimization method. J. Build. Eng. 31, 101379 (2020)

2. Y.E. Ibrahim, M. Nabil, Assessment of structural response of an existing structure under blast load using finite element analysis. Alex. Eng. J. 58(4), 1327-1338 (2019)

3. M. Yin, Y. Rui, Investigation of long-term behaviour of thermal wall by finite element analysis. Soils Found. 59(5), 1182-1192 (2019)

Publisher's Note Springer Nature remains neutral with regard to jurisdictional claims in published maps and institutional affiliations. 